\title{
Bidirectional Regulation of the cAMP Response Element Binding Protein Encodes Spatial Map Alignment in Prism-Adapting Barn Owls
}

\author{
Grant S. Nichols and William M. DeBello \\ Department of Neurobiology, Physiology and Behavior, Center for Neuroscience, University of California, Davis, Davis, California 95618
}

\begin{abstract}
The barn owl midbrain contains mutually aligned maps of auditory and visual space. Throughout life, map alignment is maintained through the actions of an instructive signal that encodes the magnitude of auditory-visual mismatch. The intracellular signaling pathways activated by this signal are unknown. Here we tested the hypothesis that CREB (cAMP response element-binding protein) provides a cell-specific readout of instructive information. Owls were fitted with prismatic or control spectacles and provided rich auditory-visual experience: hunting live mice. CREB activation was analyzed within $30 \mathrm{~min}$ of hunting using phosphorylation state-specific CREB (pCREB) and CREB antibodies, confocal imaging, and immunofluorescence measurements at individual cell nuclei. In control owls or prism-adapted owls, which experience small instructive signals, the frequency distributions of pCREB/CREB values obtained for cell nuclei within the external nucleus of the inferior colliculus (ICX) were unimodal. In contrast, in owls adapting to prisms or readapting to normal conditions, the distributions were bimodal: certain cells had received a signal that positively regulated CREB and, by extension, transcription of CREB-dependent genes, whereas others received a signal that negatively regulated it. These changes were restricted to the subregion of the inferior colliculus that received optically displaced input, the rostral ICX, and were not evident in the caudal ICX or central nucleus. Finally, the topographic pattern of CREB regulation was patchy, not continuous, as expected from the actions of a topographically precise signal encoding discrete events. These results support a model in which the magnitude of CREB activation within individual cells provides a readout of the instructive signal that guides plasticity and learning.
\end{abstract}

Key words: auditory; CREB; inferior colliculus; learning; plasticity; signal transduction

\section{Introduction}

Instructive signals are used widely to guide the development and plasticity of neural circuits. They encode behaviorally relevant information generated within one neural network that is used to fine-tune the operation of another (Knudsen, 1994). The intracellular signaling cascades that decode these well patterned signals and translate them into synaptic changes are unknown. Here we tested a novel hypothesis that activation of the cAMP response element-binding protein (CREB), a transcription factor required for several forms of learning (Kandel, 2001), encodes the magnitude of the visually based instructive signal that guides adaptive plasticity in the barn owl.

Owls are nocturnal hunters whose survival depends on auditory localization. Their brains analyze differences in the arrival time, intensity, and frequencies of sounds at each of the two ears (Konishi, 2003). These binaural cues are integrated by neurons in the external nucleus of the inferior colliculus (ICX) to form a map

Received April 1, 2008; revised July 11, 2008; accepted Aug. 1, 2008.

This work was supported by a grant from the National Institute on Deafness and Other Communication Disorders, National Institutes of Health Grant R01 DC05640 to W.M.D. and a predoctoral National Research Service Award 5F31DC008748 to G.S.N.

Correspondence should be addressed to William M. DeBello, Center for Neuroscience, University of California, Davis, 1544 Newton Court, Davis, CA 95618. E-mail: wmdebello@ucdavis.edu.

DOI:10.1523/JNEUROSCI.1385-08.2008

Copyright $\odot 2008$ Society for Neuroscience $\quad$ 0270-6474/08/289898-12\$15.00/0 of auditory space, which is relayed to the optic tectum (OT), where it merges with a map of visual space (Fig. 1A). Coalignment of these maps is essential for accurate orienting movements toward prey. Because the magnitude of binaural cues changes as the head grows or as the cochlea age, the brain must continually recalibrate map alignment. Experiments using chronic optical displacement with prismatic spectacles (Knudsen, 2002), foveal versus peripheral manipulations (Hyde and Knudsen, 2001), focal lesions in OT (Hyde and Knudsen, 2002), and pharmacological inhibition (Gutfreund et al., 2002) demonstrate that a visually based instructive signal originating in OT guides the gradual adjustment of auditory tuning in ICX. Over 2 months of prism experience, this process promotes both the strengthening of adaptive synaptic inputs and the weakening of normal, behaviorally maladaptive ones.

The molecular links between instructive signals and synaptic reorganization are unknown. Several proteins of well characterized intracellular signaling pathways are co-expressed within individual ICX cells, including CaMKII (calcium/calmodulindependent protein kinase II) (Rodriguez-Contreras et al., 2005), DARPP-32 (dopamine- and cAMP-regulated phosphoprotein with molecular weight $32 \mathrm{kDa}$ ) (Wagner et al., 2003), Cdk5 (cyclin-dependent kinase 5), MAPK (mitogen-activated protein kinase), and CREB (the latter three are reported here). Of these, CREB is an intriguing candidate because it integrates biochemical 
A

B

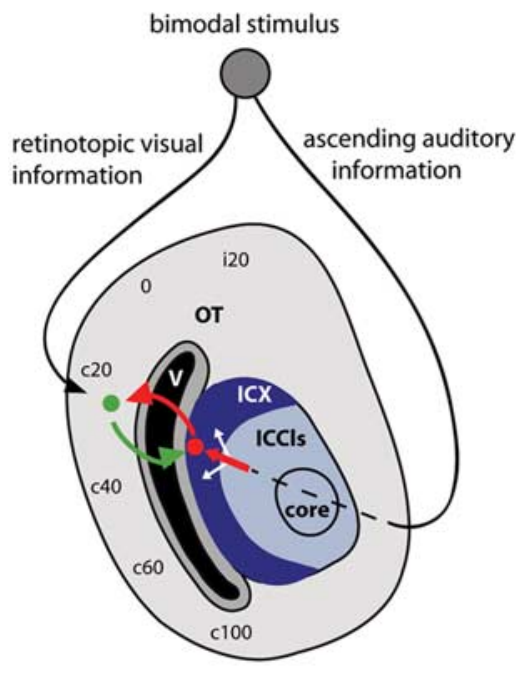

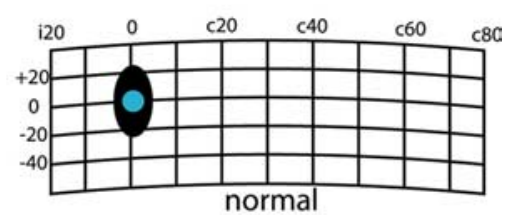
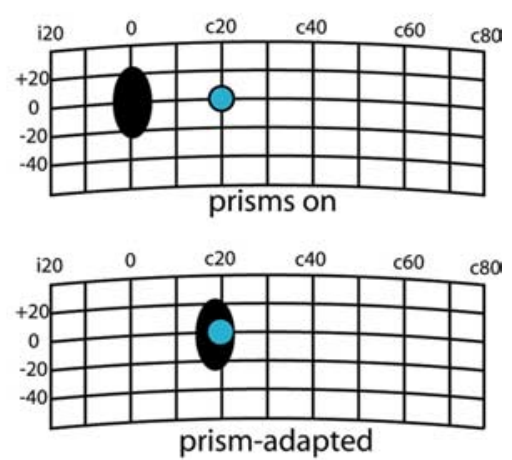
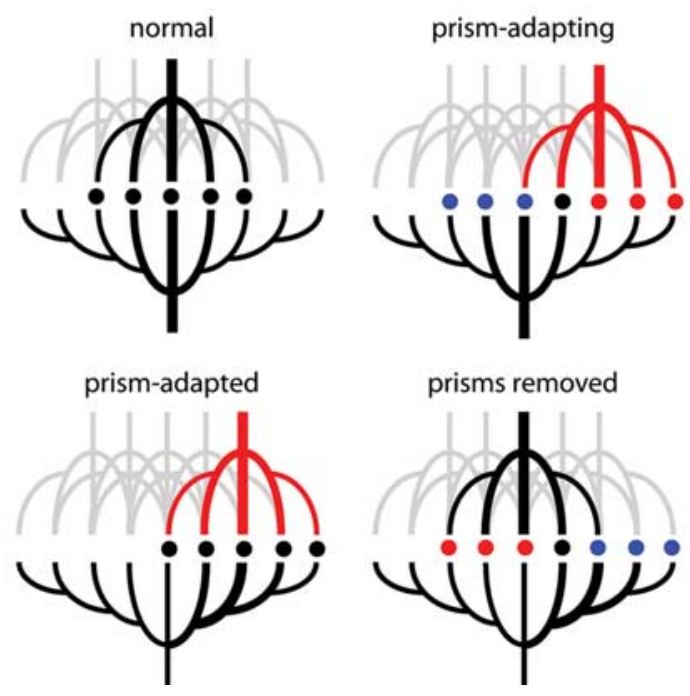

Figure 1. Instructed learning in the barn owl auditory localization pathway. $A$, Diagram of a horizontal section through the left (L) midbrain of a control owl. Ascending auditory information arrives in the core (core of the ICC) and is relayed to the ICCls (lateral shell of the ICC) on the opposite side of the brain (the contralateral pathway is not depicted). The ICCIs projects to the ipsilateral ICX, where the auditory space map is assembled. The ICCls innervates corresponding locations in ICX via topographic axonal projections, depicted by three diverging arrows. The functional strengths of connections within this broad axonal arbor are adaptively adjusted during prism experience. For control owls, the central aspect of the arbor is functionally strong (red arrow), whereas the flanks are functionally weak (white arrows). ICX neurons project to OT along a periventricular path (depicted with larger red arrow) ( $v$, ventricle), terminating in the deep layers of OT. Neurons in the deep and intermediate layers of OT receive auditory input along the serial feedforward pathway just described and also visual input via retinotopic projections. Some neurons in the intermediate and deep layers of OT project to ICX (green arrow). The OT-ICX projection is a major conduit of instructive information. $\boldsymbol{B}$ Prism-induced adaptive adjustment. Grid represents external space. Black ovals represent the auditory spatial receptive field; aqua circles represent the visual spatial receptive field for an OT neuron. Before prism mounting (normal), the fields are coaligned. Immediately after mounting prisms (prisms on), the fields are misaligned by $19^{\circ}$. After $\geq 8$ weeks of prism experience (prismadapted), the auditory field has shifted to match the optically displaced visual field. C, Schematic diagram of activity flow in the 0T for the four experimental conditions investigated in this study. Flow is diagrammed in response to a single, discrete bimodal stimulus. Visual projections are shown from above, auditory projections from below. Optically displaced visual inputs are shown in red. Functional strengths of connections are represented by line thickness. Prism adaptation involves the strengthening of auditory inputs to ICX cells receiving optically displaced inputs and the functional weakening of normal connections. Relative strengths of $A$ and $V$ inputs converging onto ICX neurons are denoted by color code: $A>V$, blue; $V>A$, red; $A \sim V$, black.

activity across all of the pathways described above and, therefore, could serve as a readout of instructive information conveyed to the cell. If this hypothesis is correct, the pattern of CREB activation in individual cells in ICX should reflect the known properties of the instructive signal (see Table 2 for list).
To investigate this hypothesis, we first cloned the owl CREB and found the predicted amino acid sequence spanning the Ser-133 phosphorylation site to be identical across several species. We then validated specificity of commercially available antibodies using in vitro biochemical assays and immunohistochemistry. The cell-specific patterns of CREB regulation were analyzed in four experimental groups reflecting distinct manipulations of the instructive signal. Within the adaptive region of ICX, the distribution of phosphorylation state-specific CREB (pCREB)/CREB values was unimodal in control owls but bimodal in adapting owls. Both the bidirectional nature and the topographic distribution of this effect reflected known aspects of the instructive signal. These data suggest a simple model in which brief episodes of behaviorally relevant experience contribute to adaptive change through bidirectional regulation of CREB.

\section{Materials and Methods \\ Animals}

Twenty barn owls (Tyto alba) were used in this study. All procedures were approved by the Institutional Animal Care and Use Committee of the University of California, Davis.

\section{cDNA cloning}

Tissue for RNA samples was prepared by brief transcardial perfusion of an owl with ice-cold $0.1 \mathrm{M}$ phosphate buffer (PB) and immediate placement of the brain in RNAlater (Invitrogen), followed by storage at $-20^{\circ} \mathrm{C}$. RNA was extracted from cerebellum using TRIzol reagent (Invitrogen). First-strand cDNA was prepared from $2.5 \mu \mathrm{g}$ RNA using the Omniscript RT kit (QIAGEN) and oligo-dT primers. Primers for PCR were designed based on regions of the CREB sequence conserved between chicken and rodents. Primer pairs used were as follows: (1) 5' -AATCTGGAGCAGAGAACCAGCA-3', 5'-TAGACGCACTTCTCTCTTCCGT-3'; and (2) 5' -AATCTGGAGCAGAGAACCAGCAGA-3', 5'ACTGAATTGCTCCTCCTTGCGT-3'. Specific products generated from PCR with these primers were visualized on a $1 \%$ agarose gel and subcloned into the pCR4-TOPO vector (Invitrogen). Sequencing was performed at the University of California, Davis DNA Sequencing Facility. A composite sequence of 819 bp was constructed by aligning the sequences generated by both sets of primers.

\section{Immunoblotting and biochemistry}

Protein samples were prepared from freshly isolated tectal lobes homogenized with a Dounce homogenizer in artificial CSF containing $1 \mathrm{mg} / \mathrm{ml}$ trypsin inhibitor and stored at $-20^{\circ} \mathrm{C}$. For immunoblot analysis, samples were suspended in SDS sample buffer, heated for $4 \mathrm{~min}$ at $95^{\circ} \mathrm{C}$, electrophoretically separated on a $12 \%$ SDS-polyacrylamide gel, and transferred to a nitrocellulose membrane. For in vitro phosphorylation, samples were incubated before suspension in sample buffer at $37^{\circ} \mathrm{C}$ for $30 \mathrm{~min}$ with $1.82 \mathrm{ng} / \mu \mathrm{l}$ PKA catalytic subunit (EMD Biosciences) and $0.73 \mu \mathrm{g} / \mu \mathrm{l}$ 
ATP. An aliquot of the phosphorylated sample was subsequently incubated for $30 \mathrm{~min}$ at $37^{\circ} \mathrm{C}$ in the presence of protein phosphatase 1 (PP-1), a phosphatase known to dephosphorylate CREB, at $20 \mathrm{ng} / \mu \mathrm{l}$ before suspension in sample buffer.

After transfer, nitrocellulose membranes were blocked for $1 \mathrm{~h}$ in $1 \times$ Odyssey blocking buffer (OD buffer; LI-COR) and then incubated for $4 \mathrm{~h}$ at room temperature in $1 \times$ OD buffer with primary antibodies at the following dilutions: mouse anti-CREB (Millipore Bioscience Research Reagents) 1:1000, rabbit anti-phospho-CREB (Cell Signaling Technology) 1:500, mouse anti-EF1 $\alpha$ (Millipore) 1:1000. After primary antibody incubation, membranes were rinsed four times for 5 min each with $0.1 \%$ Tween 20 in PBS and incubated for $1 \mathrm{~h}$ with secondary antibody diluted $1: 10,000$ in $1 \times$ OD buffer. Secondary antibodies were goat anti-rabbit 680 (Invitrogen) or goat anti-mouse 800 (LI-COR). After secondary labeling, membranes were rinsed $4 \times 5 \mathrm{~min}$ in $0.1 \%$ Tween in PBS and imaged on an Odyssey infrared scanner (LI-COR).

\section{Prism mounting}

Head bolts and prism mounts were surgically attached to the owl's skull. Anesthesia and postoperative care were provided according to University of California, Davis Veterinary Care standards. Details of the surgical procedure can be found in previous articles (Swofford and DeBello, 2007; McBride et al., 2008). Surgeries were performed at $\sim 65 \mathrm{~d}$ of age. After recovery from surgery, owls were released into large, outdoor group aviaries for 1 week before prism mounting. During this time, they engaged in post-fledging behaviors: mastery of flight and hunting live mice. Subsequently, $19^{\circ}$ right-shifting Fresnel lenses (prisms) or optically transparent lenses (control) were set in spectacle frames and secured to the prism mount previously attached to the skull. A complete summary of the experimental groups is given below.

\section{Experimental groups}

Control (cases 1, 3, 5, 7, 9, 11). Six juvenile owls were fitted with optically transparent, nonshifting lenses. Owls were perfused at 75-90 d, after $48-72 \mathrm{~h}$ of experience with control glasses in place. Case 11 was an age-matched control for cases 13-16 (ages at perfusion, 150-160 d). All control owls experienced small auditory-visual mismatches during the final hunting episode.

Prism-adapting (cases 2, 4, 6, 8, 10, 12). Six juvenile owls were fitted with $19^{\circ}$ right-shifting lenses. Owls were perfused at 75-90 d, after $48-72$ hours of prism experience. Case 12 was an older prism-adapting owl for comparison with owls 11,13,14,15, and 16 (ages at perfusion, 150-180 days). All prism-adapting owls experienced large auditory-visual mismatches during the final hunting episode.

Prism-adapted (cases 13 and 14). Two juvenile owls were fitted with $19^{\circ}$ right-shifting lenses at age $\sim 70 \mathrm{~d}$ and released into flight aviaries for $>2$ months of prism experience. Adaptive shifts were confirmed at age 130$140 \mathrm{~d}$ by electrophysiological mapping. Owls were perfused at 150-180 d. All prism-adapted owls experienced small auditory-visual mismatches during the final hunting episode.

Prism-removed (cases 15 and 16). Two juvenile owls were raised according to the conditions described above for prism-adapted owls. Their prisms were removed $24 \mathrm{~h}$ before perfusion. All prism-removed owls experienced large auditory-visual mismatches during the final hunting episode.

\section{Hunting}

All owls used in this study were juveniles within the sensitive period for prism adaption, i.e., <200 d (Brainard and Knudsen 1998). The durations of prism-rearing and ages of killing for the four experimental groups are described above. For hunting, prism-adapting and control owls or prism-removed and prism-adapted owls were grouped in pairs. One at a time, pairs were isolated in an aviary containing elevated perches that allowed them to survey the hunting ground, an $8^{\prime}$ by $16^{\prime} \times 14^{\prime \prime}$ (width $\times$ length $\times$ depth) walled zone located in the center of the aviary. The floor of the hunting ground was covered with a thin layer of straw. After $2 \mathrm{~d}$ of fasting, prism or control glasses were mounted and live mice were released into the hunting ground. Mice were visible, moving along the surface of the straw, and their movements generated localizable sounds. Retrospective videography with an infrared (night vision) at- tachment confirmed that owls hunted within 10 min of the release of the mice into the hunting ground (range, 1-10 min). To maintain incentive for rapid hunting, owls fasted for two additional days before the final session. Thus the total duration of prism experience for prism-adapting owls was $3 \mathrm{~d}$. This duration results in very little, if any, adaptive change in auditory-visual map alignment (DeBello and Knudsen, 2004). Therefore, the auditory-visual mismatches experienced by these owls during the final hunting session must have been very close to the full optical displacement imposed by the prisms, $19^{\circ}$.

Thirty minutes after release of the mice into the hunting ground, owls were captured and promptly anesthetized with $5 \%$ isofluorane in $1 \mathrm{~L} / \mathrm{min}$ $\mathrm{O}_{2} / \mathrm{NO}_{2}$. Blood was cleared by transcardial perfusion with $0.1 \mathrm{M} \mathrm{PB}$ containing $3 \mathrm{cc} / \mathrm{L}$ bupivicaine, and the animal was perfused with $\sim 700 \mathrm{ml}$ of fixative solution ( $4 \%$ paraformaldehyde in $0.1 \mathrm{M} \mathrm{PB}$ ) followed by fixative containing $10 \%$ sucrose. After being allowed to fix in situ for $2 \mathrm{~h}$, the brain was removed and cryoprotected in $30 \%$ sucrose in $0.1 \mathrm{M} \mathrm{PB}$ at $4^{\circ} \mathrm{C}$.

\section{Immunohistochemistry}

Horizontal sections ( $40 \mu \mathrm{m}$ thick) were prepared with a freezing microtome. Sections were blocked at room temperature $\left(R_{T}\right)$ for $1.5 \mathrm{~h}$ in 0.1 M PB containing 4.0\% normal goat serum (Vector Laboratories), 1.0\% bovine serum albumin (ThermoFisher Scientific), and $0.4 \%$ Triton $\mathrm{X}-100$. Primary antibodies were diluted in $0.1 \mathrm{M} \mathrm{PB}$ containing $1.0 \%$ normal goat serum, $1.0 \%$ bovine serum albumin, and $0.3 \%$ Triton X-100 as follows: mouse anti-CREB (Millipore Bioscience Research Reagents), 1:500 or 1:100,000 (see below); rabbit anti-phospho-CREB (Cell Signaling Technology), 1:50; rabbit anti-pMAPK, 1:200; rabbit anti-cdk-5 (Santa Cruz Biotechnology), 1:100; mouse anti-DARPP-32 (gift from H. C. Hemmings, Weill Cornell Medical College, New York, NY), 1:5000; mouse anti-CaMKII $\alpha$ (Millipore Bioscience Research Reagents), 1:650 or 1:100,000 (see below); rabbit anti-calbindin (Swant), 1:1000; rabbit anti-calretinin (Swant), 1:1000; mouse anti-NeuN (Millipore Bioscience Research Reagents), 1:100,000, and incubated overnight at $4^{\circ} \mathrm{C}$. Sections were rinsed twice for 5 min each in $0.02 \%$ Triton X-100 and $0.25 \%$ BSA in $\mathrm{PB}$ at $\mathrm{R}_{\mathrm{T}}$. Secondary antibodies were obtained from Invitrogen. Secondary antibodies were diluted at 1:1000 in a solution containing $0.02 \%$ Triton X-100 and $0.25 \%$ BSA in PB, and the incubation proceeded for $1.5 \mathrm{~h}$ at $\mathrm{R}_{\mathrm{T}}$. After incubation with secondary antibody, sections were rinsed five times for $2 \mathrm{~min}$ each in $0.25 \% \mathrm{BSA}$ in $\mathrm{PB}$ and then twice for 5 min each in PB. Sections were then mounted out of $0.3 \%$ gelatin in $\mathrm{dH}_{2} \mathrm{O}$ on glass coverslips. After drying, coverslips were mounted on glass slides in Vectashield Hardset (Vector Laboratories) and sealed with nail polish. A processing group consisted of tissue from up to four animals that were processed concurrently using common solutions. In cases in which antibodies of the same isotype were used together (such as CaMKII $\alpha$ and CREB, or NeuN and CREB), one antibody (CaMKII $\alpha$ or NeuN) was used at very low concentration $(1: 20,000-1: 100,000)$ and amplified with a Tyramide Signal Amplification (TSA) kit (Invitrogen) used according to the manufacturer's instructions, followed by incubation with the CREB antibody and detection using conventional fluorescent secondary antibodies as described above.

\section{Image acquisition and analysis}

Images were acquired from both the left and right tectal lobes of experimental animals. Sections near the dorsoventral level of the optic ventricle were selected. The ICX was distinguished from the superficial nucleus of the inferior colliculus by examination of autofluorescence through a green filter. Accuracy in identifying ICX was verified in several cases in which adjacent sections were labeled with anti-CaMKII $\alpha$, which selectively labels ICX (Rodriguez-Contreras et al. 2005). The location of the central nucleus of the inferior colliculus (ICC) was estimated based on the distance from ICX. The location of each image field in ICX or ICC was noted on a sketch of the section. The rostrocaudal locations of image fields were measured relative to a line drawn parallel to the medial boundary of the inferior colliculus, stretching from the rostral to the caudal end. The metric ranged from 0 to 1.0, with 1.0 being most rostral.

Images were obtained on a Zeiss 510 META confocal microscope system using a Zeiss Plan-APOCHROMAT $63 \times 1.4$ numerical aperture lens. The phospho-CREB antibody was detected using an anti-rabbit 
Table 1. Case summary of CREB phosphorylation patterns

\begin{tabular}{|c|c|c|c|c|c|c|c|c|}
\hline \multirow[b]{2}{*}{ Comparison } & \multirow[b]{2}{*}{ Prism } & \multirow[b]{2}{*}{ Control } & \multirow[b]{2}{*}{$\Delta$ Instructive Signal } & \multirow[b]{2}{*}{ Age } & \multirow[b]{2}{*}{ Duration } & \multicolumn{3}{|c|}{ Shape change vs control } \\
\hline & & & & & & $\mathrm{rICX}$ & $\mathrm{CICX}$ & ICC core \\
\hline 1 & $\mathrm{R} 19^{\circ}$ & $0^{\circ}$ & $19^{\circ}$ & $75-90 d$ & $\sim 72 \mathrm{~h}$ & Bidirectional & Lateral shift & No change \\
\hline 2 & $\mathrm{R} 19^{\circ}$ & $0^{\circ}$ & $19^{\circ}$ & $75-90 d$ & $\sim 72 \mathrm{~h}$ & Bidirectional & No change & n.d. \\
\hline 3 & $\mathrm{R} 19^{\circ}$ & $0^{\circ}$ & $19^{\circ}$ & $75-90 d$ & $\sim 72 \mathrm{~h}$ & Bidirectional & No change & Lateral shift \\
\hline 4 & $\mathrm{R} 19^{\circ}$ & $0^{\circ}$ & $19^{\circ}$ & $75-90 d$ & $\sim 72 \mathrm{~h}$ & Bidirectional & Lateral shift & n.d. \\
\hline 5 & $\mathrm{R} 19^{\circ}$ & $0^{\circ}$ & $19^{\circ}$ & $75-90 d$ & $\sim 72 \mathrm{~h}$ & Lateral shift & No change & No change \\
\hline 6 & $\mathrm{R} 19^{\circ}$ & $0^{\circ}$ & $19^{\circ}$ & $150-180 \mathrm{~d}$ & $\sim 24 \mathrm{~h}$ & Bidirectional & Lateral shift & No change \\
\hline 7 & Removed & Adapted & $16.3^{\circ}$ & $150-180 d$ & $\sim 24 \mathrm{~h}$ & Bidirectional & Bidirectional & Lateral shift \\
\hline 8 & Removed & Adapted & $14.1^{\circ}$ & $150-180 d$ & $\sim 24 \mathrm{~h}$ & Bidirectional & Lateral shift & No change \\
\hline 9 & Removed & $0^{\circ}$ & $18.7^{\circ}$ & $150-180 d$ & $\sim 24 \mathrm{~h}$ & Bidirectional & Bidirectional & Lateral shift \\
\hline 10 & Removed & $0^{\circ}$ & $16.5^{\circ}$ & $150-180 d$ & $\sim 24 \mathrm{~h}$ & Bidirectional & Lateral shift & No change \\
\hline 11 & Adapted & $0^{\circ}$ & $0.7^{\circ}$ & $150-180 d$ & $>60 d$ & No change & Lateral shift & No change \\
\hline 12 & Adapted & $0^{\circ}$ & $4.1^{\circ}$ & $150-180 d$ & $>60 d$ & No change & No change & No change \\
\hline
\end{tabular}

Case-to-case comparisons of prism-adapting/prism-removed owls and their cognate controls. The comparison numbers do not correspond to the case numbers listed in Materials and Methods, Experimental groups. "Prism" indicates the status of the experimental animal: R19 (prism-adapting), removed (prism-removed), and adapted (prism-adapted). "Control" indicates the status of the control owl: $0^{\circ}$ (clear lenses) or adapted. " $\Delta$ Instructive Signal" indicates the approximate magnitude of the instructive signal experienced during the final hunting episode, computed by subtracting the value in the cognate control from the known (19 ${ }^{\circ}$ for prism-adapting) or measured (determined electrophysiologically for prism-adapted and prism-removed owls) value in the experimental animal. Bold text indicates cases in which a large A-V mismatch was experienced. "Duration" indicates length of time in the final condition being tested. "Shape change vs control" indicates the difference in pCREB/CREB distribution between experimental and control animals. Bold text indicates cases in which bidirectional CREB regulation was observed. n.d., Not determined.

antibody conjugated to AF 568 (Invitrogen); the CREB antibody, using an anti-mouse antibody conjugated to AF 647. Images were acquired in multi-track mode to eliminate cross talk between channels. The AF 568 fluorophore was excited with a $\mathrm{HeNe} 543 \mathrm{~nm}$ laser line, and the signal was collected using a 565-614 nm bandpass filter. The AF 647 fluorophore was excited using the HeNe $633 \mathrm{~nm}$ laser line and the signal was collected without a filter. As expected from the excitation and emission spectra of the fluorophores, no cross-channel bleed-through was observed (data not shown).

Laser intensity and detector gain were adjusted before image acquisition to use full dynamic range. Image stacks were analyzed using Volocity (Improvision). Nuclei were classified by co-application of minimum intensity and volume $\left(10 \mu \mathrm{m}^{3}\right)$ thresholds applied to the CREB image (all $\mathrm{pCREB}+$ nuclei were CREB + but not vice versa). The average intensities of CREB and pCREB staining within classified volumes were measured and stored as primary data.

Because detector gain was adjusted on a per field basis, absolute intensity values were not always comparable between image stacks. Primary data were transformed using gain response functions derived from measuring the intensity of fluorescent beads (InSpeck; Invitrogen) at similar gain settings and identical laser power and filter settings (supplemental figure, available at www.jneurosci.org as supplemental material). Before intensity correction, the background staining intensity for each channel was measured and subtracted from the PCREB and CREB intensities. Background staining intensity was measured in an area containing no distinguishable biological structure. The corrected values for PCREB and CREB at each nucleus were used for subsequent analyses.

\section{Quantitative analysis of $p C R E B / C R E B$ distributions}

Frequency histograms of pCREB/CREB values were constructed independently for each case (16 owls) and region within (rostral ICX, caudal ICX, ICC). Outliers were removed (lowest and highest $2.5 \%$ of values), and the remaining range was normalized from 0 to 1 based on the lowest and highest values observed throughout the processing group. This procedure did not cause a relative shift or shape change in the distributions for within-group comparisons. Most comparisons, including all of those presented in Table 1, are within-group comparisons. The exceptions are summary data shown in Figure $4 B$, right panel, and Figure 5. These data were constructed by first normalizing individual cases to peak (one unit) and then averaging the relevant cases.

Statistical comparisons between distributions were made using the Kolmogorov-Smirnoff test. The $p$ value reflects the maximum divergence of the cumulative probability plots. To test the significance of bidirectional effects, distributions were clipped at their inflection point. The resultant plots effectively isolated either the lower or upper region, respectively, of actual data for statistical analysis. For example, for the comparison shown in Figure $4 B$, left panel, the divergence on both sides of the inflection point is highly significant $(p<0.0001$; classified as a "bidirectional" shift in Table 1). In other cases, the comparison was significant in only one direction (classified as a "lateral" shift) or insignificant in both directions (classified as "no change").

\section{Electrophysiology}

Details of the electrophysiological procedure can be found in previous work (Rodriguez-Contreras et al., 2005; Swofford and DeBello, 2007). Briefly, owls were subdued with $\mathrm{NO}_{2} / \mathrm{O}_{2}$ and secured inside an acoustic recording chamber. Auditory-visual alignment was assessed using multiunit recording (Thomas Recording) from the deep and intermediate layers of OT. Stimulus generation and data acquisition were controlled by customized software written by A. Collins (Spike2; Cambridge Electronic Design).

To determine auditory-visual alignment, the azimuthal coordinate of the visual receptive field (Vrf) was determined (with prisms removed) by projecting a visual stimulus onto a tangent screen. The azimuthal coordinate of the auditory receptive field was determined by presenting broadband noise bursts through an array of 16 speakers positioned in front of the owl (Spike2; Cambridge Electronic Design). The stimuli were presented 20-30 times per speaker, and the auditory best location was computed as the weighted average of responses to stimuli at the individual speakers. The prism-induced adaptive shift for each multi-unit was computed as the difference between the Vrf and the auditory best location.

\section{Results}

The goal of this study was to test the hypothesis that CREB regulation encodes the magnitude of auditory-visual mismatch. We examined four groups of juvenile owls representing different stages of adaptation: owls wearing control glasses (control), owls with recently mounted prisms (prism-adapting), owls adapted to prisms (prism-adapted), prism-adapted owls with prisms recently removed (prism-removed).

\section{CREB, CaMKII, DARPP-32, CDK-5, and MAPK are strongly expressed within ICX neurons}

Our hypothesis was motivated in part by the observation that neurons in the ICX express several well characterized intracellular signaling proteins that activate in response to synaptic stimulation (Fig. 2). Many proteins were coexpressed within individual neurons, including CaMKII and DARPP-32 (Fig. 2B,C) and CaMKII and CREB (Fig. 3C). Because all of these signaling pathways converge at the level of a single molecule, CREB, the observations suggest a model in which information conveyed by the instructive signal is integrated by CREB, resulting in its cell- 


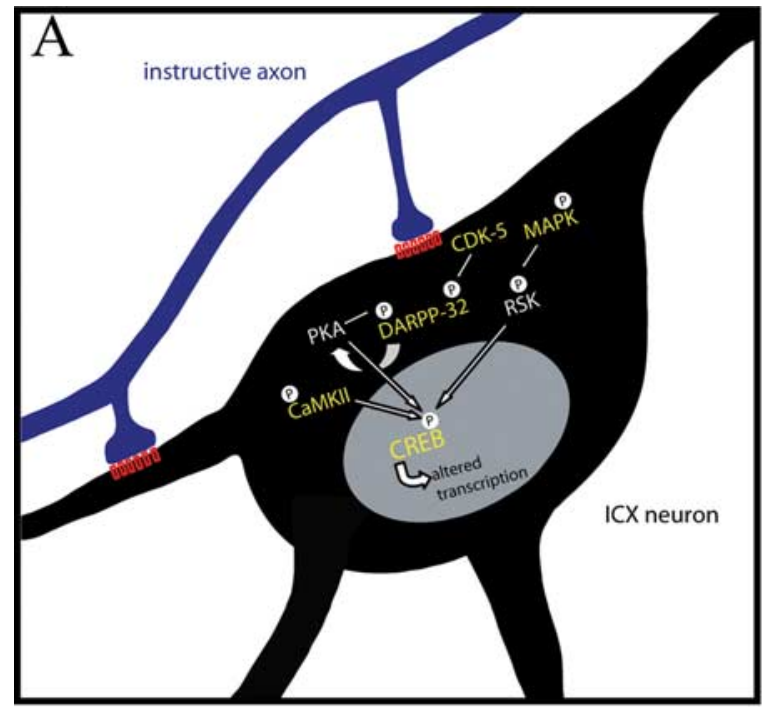

$\mathrm{B}$

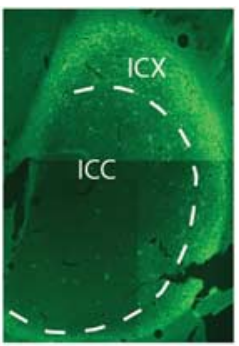

$\mathrm{C}$
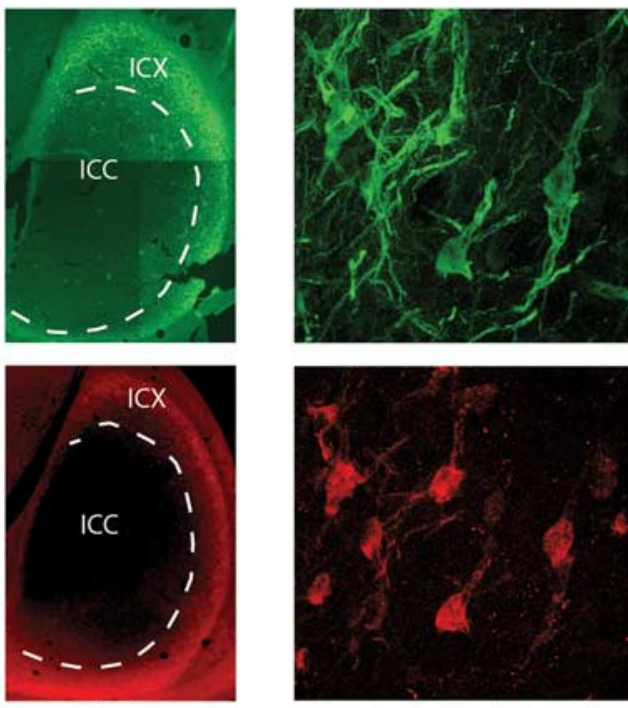

-
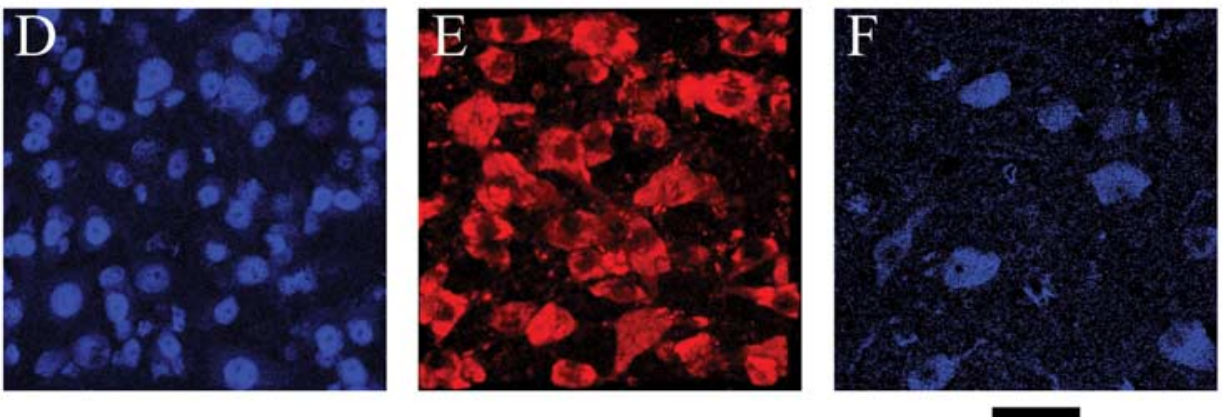

Figure 2. CREB-related intracellular signaling proteins expressed in ICX.A, Illustration of an ICX neuron receiving synaptic contact from an instructive axon (OT-ICX) projection. The postsynaptic receptor(s) at this synapse have not been identified, but five intracellular signaling pathways that commonly act downstream of synaptic transmission are strongly expressed in owl ICX-MAPKII, Cdk-5, DARPP-32, CaMKIII, and CREB. B, CaMKII expression (green) was mainly restricted to ICX ( $C$, left; scale bar (for $\boldsymbol{B}, \boldsymbol{C}), 500 \mu \mathrm{m}$ ) and distributed throughout somatodendritic compartments (C, right; scale bar (for $B, C), 40 \mu \mathrm{m}$ ). C, DARPP-32 expression (red) was mainly restricted to ICX (left) and found in cell bodies and puncta. Individual cell bodies that expressed CaMKII or DARPP-32 usually expressed both, as shown by the correspondence of cells in $\boldsymbol{B}$ and $\boldsymbol{C}$, which are two channels from the same confocal image stack. $\boldsymbol{D}$, (REB expression (blue) was confined to nuclei that occurred at a high spatial density. In limited triple-labeling experiments, all CaMKII + cells also expressed CREB ( $n=87)$. $\boldsymbol{E}-\boldsymbol{F}$, MAPK expression (red) $(\boldsymbol{E})$ and (DK-5 expression (blue) $(\boldsymbol{F})$ were mainly perisomatic.

specific net phosphorylation or dephosphorylation. We developed tools to test this model.

\section{Characterization of barn owl CREB cDNA and protein}

We cloned a partial cDNA corresponding to the owl homolog of CREB. The 819 bp sequence exhibited 94,85 , and $85 \%$ nucleotide identity to zebra finch, human, and mouse, respectively. The predicted amino acid sequence spanning the Ser-133 phosphorylation site was identical across species (Fig. $3 A$ ).

To determine the utility of commercially available antibodies in monitoring changes in CREB activation, we used immunoblotting and in vitro phosphorylation. Antibodies directed against either CREB protein (CREB) or a phosphorylated synthetic peptide (pCREB) recognized a band at the expected molecular weight of $43 \mathrm{kDa}$ on blots of owl brain protein. Additional faint bands were observed, and this pattern of staining was indistinguishable from blots prepared with mouse brain protein (not shown). Preincubation of the owl sample with protein kinase A, which directly phosphorylates CREB, increased the intensity of pCREB staining (Fig. 3B), and treatment with PP-1, known to dephosphorylate CREB (Hagiwara et al. 1992), reversed this effect. Neither of these in vitro reactions altered the intensity of staining with the CREB antibody (data not shown). These data demonstrate that the pCREB antibody can be used in owl to track changes in phosphorylation state.

Quantitative Western blotting is widely used to measure CREB phosphorylation by analyzing the relative staining intensity produced by $\mathrm{pCREB}$ and CREB antibodies (pCREB/CREB). The disadvantage of this technique is that valuable information regarding cell-to-cell variability is lost. To circumvent this limitation, we analyzed pCREB/CREB in fixed sections of the owl midbrain using immunohistochemistry, confocal microscopy, and three-dimensional image analysis. As expected, CREB was expressed almost exclusively in cell nuclei (Figs. $2 D, 3 C, D$ ) in all brain structures examined: the ICX, ICC, and OT. The cellular pattern of staining for pCREB was strikingly similar with very strong correspondence between color channels (Fig. 3C). These results, in combination with the in vitro biochemical data, indicate that the ratios of pCREB/CREB intensity within individual cell nuclei reflect the activation states of CREB at the time of killing.

\section{Bidirectional regulation of CREB in the rostral ICX of} prism-adapting fledglings

Newly fledged owls (75-90 d) were fitted with prism or control glasses and required to hunt live mice. CREB phosphorylation 
A

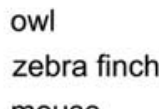

mouse

human

C

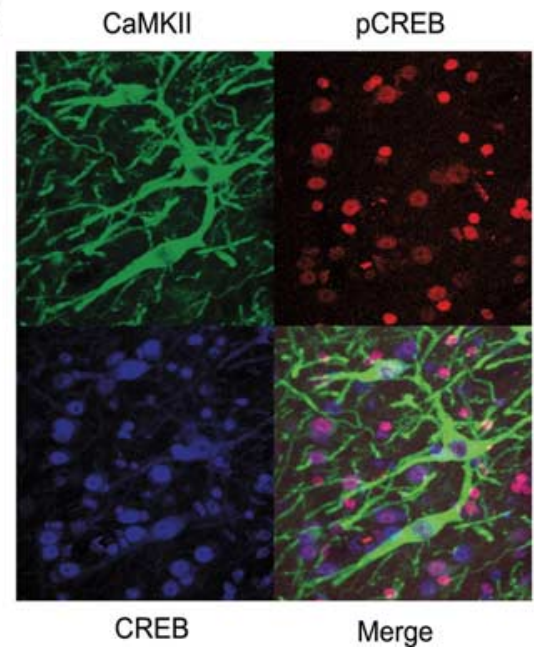

110- RREILSRRPSYRKILNDLSSD

110- RREILSRRPSYRKILNDLSSD

110- RREILSRRPSYRKILNDLSSD

110- RREILSRRPSYRKILNDLSSD

$-130$

$-130$

$-130$

$-130$

D

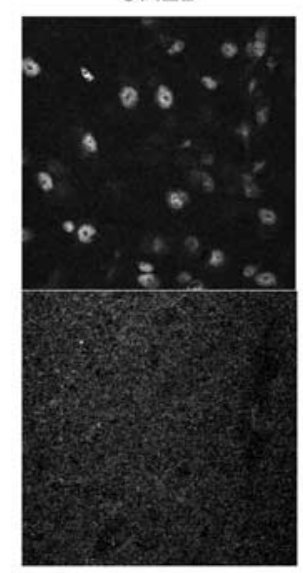

pCREB + peptide
B

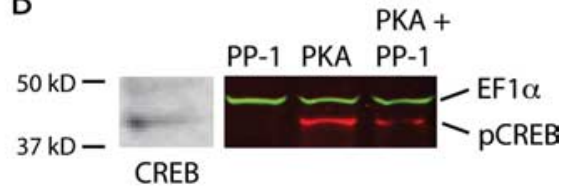

E

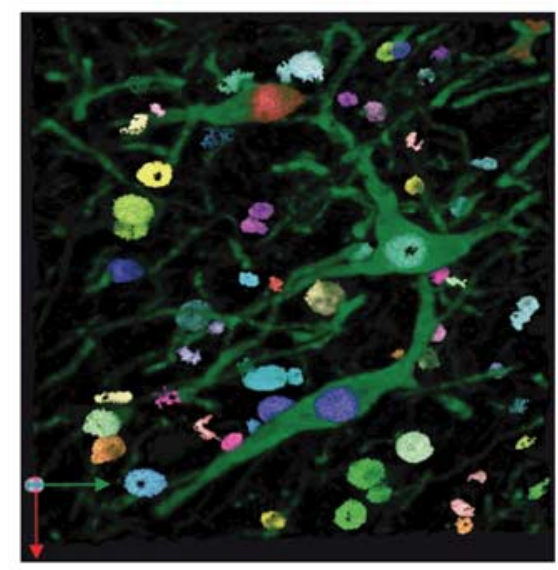

Figure 3. Cloning, in vitro phosphorylation, and immunofluorescent detection of barn owl CREB. A, Deduced amino acid sequence for owl CREB and its cross-species alignment. The sequence surrounding the phosphorylation site is $100 \%$ conserved. In the isoform we cloned, CREB327 derived from the CREB1 gene, the serine residue occurs at position 119 . It is functionally analogous to serine 133 of the CREB341 isoform. $\boldsymbol{B}$, Western blots prepared with owl brain homogenates and probed with CREB (left) or pCREB (right) antibodies. PP-1 preincubation eliminated all pCREB staining (red bands). Preincubation with phosphokinase A, which specifically phosphorylates serine 119/133, produced intense labeling, which was mostly reversible by subsequent treatment with PP-1. Green bands reflect detection of EF1 $\alpha$, a protein-loading control. C, Triple-labeled section with CaMKII (green), pCREB (red), and CREB (blue). D, Preincubation of pCREB antibody with the antigenic peptide eliminated pCREB but not CREB immunodetection in tissue labeled with both antibodies. Each antibody was detected on a separate color channel. E, Automated classification of CREB nuclei applied to the image shown in C. Each nucleus is rendered in a different pseudo-color. pCREB and CREB intensity measurements were made within these volumes.

patterns were analyzed on day 3 . The accumulated experience over this time does not cause significant adjustment of the auditory map, and therefore, these owls experienced large auditoryvisual mismatches even during the final hunting episode.

To analyze CREB activation at the conclusion of this episode, owls were perfused with paraformaldehyde $30 \mathrm{~min}$ after release of mice into the hunting area, and brain sections were prepared for immunohistochemistry and confocal analysis. Frequency distributions of pCREB/CREB values for a representative prismadapting and control owl are shown in Figure $4 \mathrm{~B}$. Within rostral ICX, a region that represents frontal space, the control distribution was unimodal and Gaussian in shape, with a positive skew. In contrast, the prism-adapting distribution was bimodal, with many of the individual values pushed closer to the lower or upper boundaries of the distribution. This change in shape was highly significant $(p<0.0001$, Kolmorogov-Smirnoff test) for both populations of nuclei: i.e., ones that exhibited increase or decreases. We refer to this effect hereafter as bidirectional regulation of CREB. Similar results were observed with four of the five pairs of prism and control juveniles (cumulative probability plots; Fig. $4 B$ right panels, insets) and were evident in data pooled across individuals $(p<0.0001$; cumulative probability plots, Fig. $4 B$ bottom panels, main graphs).

\section{CREB regulation in caudal ICX and ICC}

We next examined CREB regulation in two additional regions, the caudal ICX and ICC. Caudal ICX represents peripheral space, a region not reliably exposed to optical displacement because of the physical design of the surgically mounted prism lenses. Consequently, the caudal aspects of ICX do not typically experience large auditory-visual mismatches. The distribution of pCREB/
CREB values observed in the caudal ICX of prism-adapting owls was unimodal, and similar in shape to that observed in the caudal ICX of control owls (Fig. 5A). Likewise, the ICC does not receive instructive information and is not a site of prism-induced plasticity. The pCREB/CREB distributions in ICC were very similar between prism-adapting and control owls (Fig. 5B). In summary, the only locations within the inferior colliculi of either prismadapting or control owls that exhibited strikingly altered shape distributions were the ones that received large-magnitude instructive signals (i.e., rostral ICX).

\section{CREB regulation in prism-adapted owls}

Six owls, two with control glasses and four with prisms, were raised at least $60 \mathrm{~d}$ post-fledging, sufficient time for full adaptation, which manifests as the appearance of responses to sound locations that match the optically displaced visual field location, and the suppression of responses corresponding to the visual field location with prisms removed. We measured the magnitude of adaptive shifts in each animal using multi-unit recording from the OT. A representative raster plot is shown in Figure 6A. The weighted average of auditory tuning relative to visual receptive field location with prisms removed is shown in Figure $6 B$ for the population of recording in this animal and three others. Mean adaptive shifts ranged from 15.0 to $18.7^{\circ}$. These values are close to the $19^{\circ}$ optical displacement imposed by the Fresnel lenses and are typical in magnitude of fully adapted owls. More importantly, the auditory-visual mismatch experienced by each of these animals at the time of mapping was small. After recovery from mapping, prisms were left in place for two fully adapted owls (prismadapted, cases 13 and 14) but removed from two others (prismremoved, cases 15 and 16). The latter group now experienced 
A

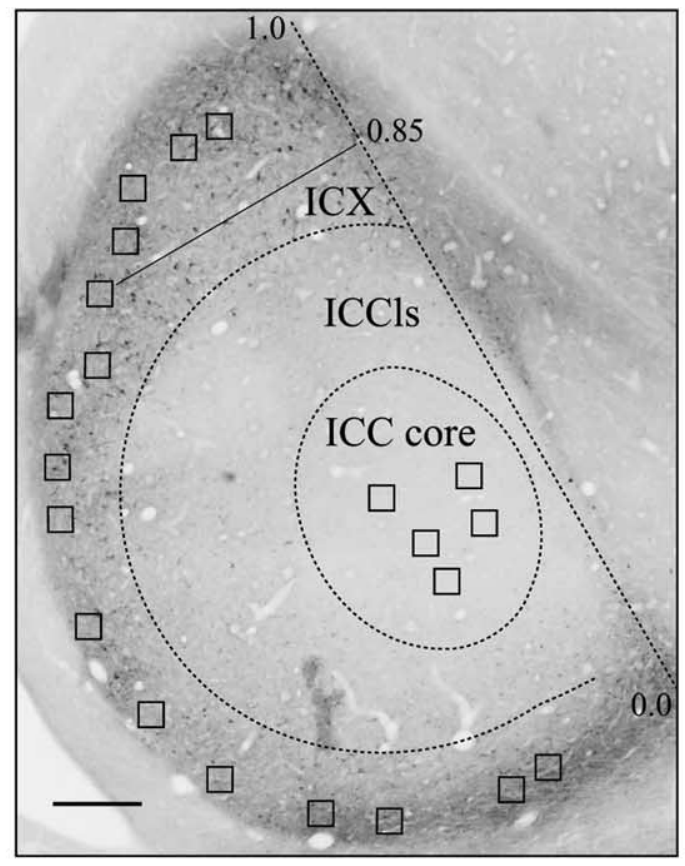

$\mathrm{B}$
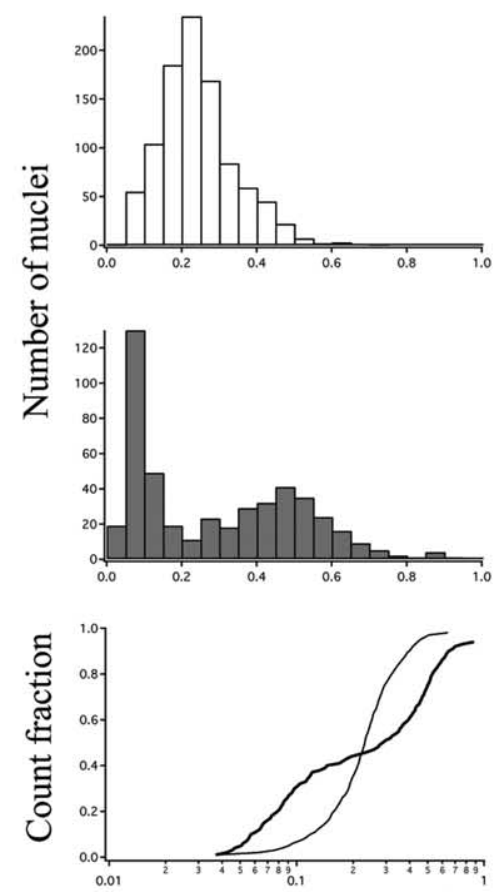

all cases
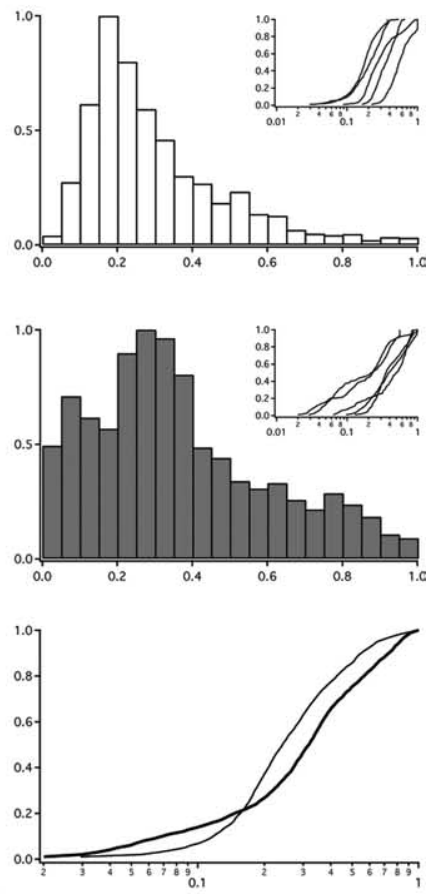

pCREB/CREB

Figure 4. Prism-induced bidirectional CREB regulation in rostral ICX. $A$, Representative section used for analysis. Immunofluorescent images were obtained at lower magnification (5X objective), montaged, and converted to grayscale for display. Visible staining is for CaMKII, which delineates the boundaries of ICX. The rostral (1.0) and caudal ( 0.0$)$ poles of ICX are indicated along the medial border. The location of the ICC core is estimated. Small boxes in the ICX and ICC core represent locations and sizes of the high-magnification image fields used for CREB and pCREB analysis on the adjacent sections labeled with these antibodies. The rostrocaudal location of these image fields in ICX was determined from orthogonal projections as the fractional distance from the caudal to the rostral pole (i.e., 0.85 for the fifth most rostral field). Scale bar, $500 \mu \mathrm{m}$. B. Frequency histograms of pCREB/CREB values measured in individual nuclei for control (open bars) and prism-adapting (gray bars) owls. This analysis was restricted to image fields at rostrocaudal locations between 0.4 and 1.0 (rostral ICX). The bottom panels are cumulative probability plots of the data shown directly above (control owls, thin black line; prism-adapting owls, thick black line). The left panels show results for an exemplar control and prism-adapting owl. Numbers of nuclei are displayed as absolute counts. The right panels show data combined for all young juvenile control $(n=5)$ and prism-adapting $(n=5)$ owls. Numbers of nuclei are normalized to peak. Shown in the small insets are the cumulative probability plots for each individual owl. The plots for all five control cases were comparably smooth and sigmoidal in shape. In contrast, the plots for all five prism-adapting cases were stretched horizontally and irregular in shape.

large auditory-visual mismatches. All four owls were returned to the aviaries for a final hunting episode.

CREB activation patterns for prism-adapted and prismremoved owls are shown in Figure 7. In prism-adapted owls, pCREB/CREB distributions were unimodal within rostral ICX, caudal ICX, and ICC. No bidirectional regulation was observed. These patterns recapitulate those observed in control owls. In contrast, in prism-removed owls, the distributions were bimodal in rostral ICX and unimodal in ICC. These patterns recapitulate those observed in prism-adapting owls. For case 15, the distribution in caudal ICX was unimodal, also consistent with previous findings. For case 16 there was a clear bidirectional effect evident in caudal ICX (Fig. 7B).

Finally, prisms were mounted on one of the two post-fledging control owls at age $165 \mathrm{~d}$, and CREB patterns were analyzed after one hunting episode in this owl and the remaining control. pCREB/CREB distributions for this pair were comparable to those observed for younger juveniles, ages 75-90 d (data not shown).

In summary, across all owls in which CREB activation was analyzed, there was a strong correlation between the magnitude of auditory-visual mismatch and the extent of CREB regulation (Table 1). Within rostral ICX, seven of eight owls experiencing large auditory-visual mismatches exhibited bidirectionally shifted CREB distributions (relative to cognate controls), whereas zero of two experiencing small auditory-visual mismatches did. Within caudal ICX, the corresponding results were one of eight and zero of two. Within ICC, the results were zero of eight and zero of two.

\section{Topographic pattern of bidirectional CREB regulation}

We next examined the topographic pattern of bidirectional CREB regulation. One possibility is that $\mathrm{pCREB} / \mathrm{CREB}$ values were similar between image fields, indicative of a continuous distribution. This would result from a global process acting throughout rostral ICX. The other possibility is that some image fields contained nuclei expressing, on average, small pCREB/ CREB values, whereas others contained nuclei expressing large ones. Such a patchy distribution would result from the actions of an instructive signal encoding discrete events.

The distribution of all pCREB/CREB values measured in the rostral ICX of a single adapted owl (case 13) is shown in Figure $8 A$, top left panel. The mean value for each field was similar, regardless of topographic location (Fig. $8 \mathrm{~A}$, filled circles, bottom left panel), and this was true of all owls experiencing smallmagnitude auditory-visual mismatches (Fig. $8 A$, open circles, bottom left panel). The analogous data for a prism-removed owl (case 15) and all owls experiencing large auditory-visual mismatches are shown in Figure $8 \mathrm{~A}$, right. There was considerable field-to-field variation. To quantify this effect, we measured the 


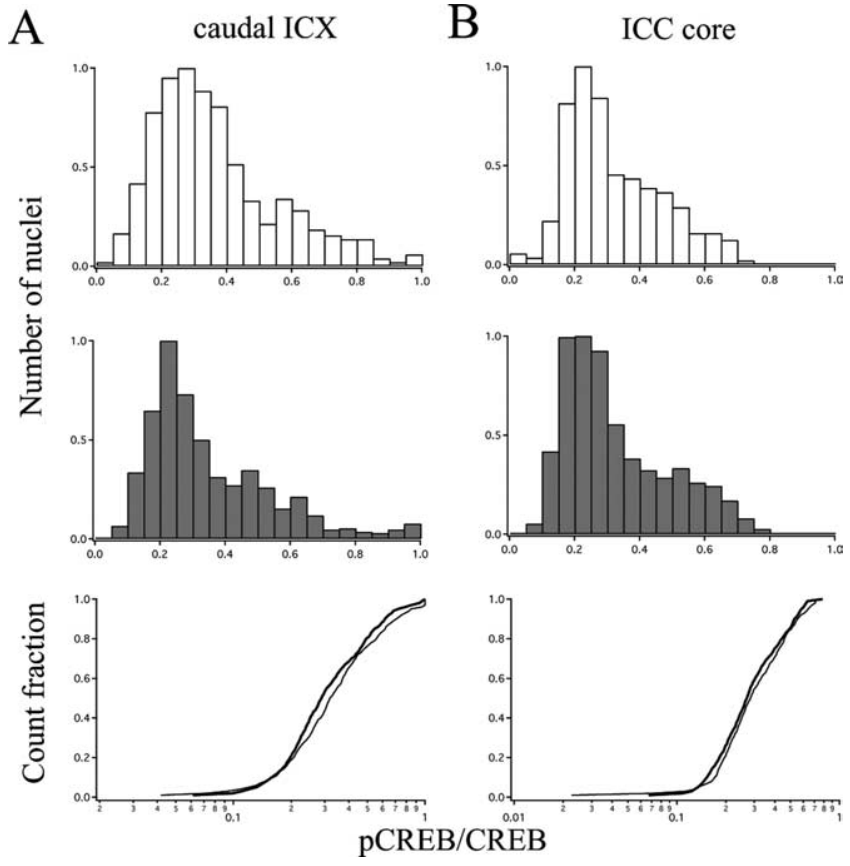

Figure 5. $A, B$, Minimal CREB regulation in caudal ICX or ICC. $p$ CREB/CREB distributions for image fields located in caudal ICX ( $A$; at rostrocaudal locations between 0.0 and 0.4 ) or ICC core $(B)$. All other aspects of this analysis are as described in the Figure 4 legend. In caudal ICX, the cumulative probability plot (bottom panel) for prism-adapting cases was shifted slightly to the left compared with control cases $(p=0.003)$. In ICC, the cumulative probability plots were nearly overlapping $(p=0.26)$.

difference between mean pCREB/CREB values from one image field to its nearest neighbor. The distribution of these difference values is shown in Figure $8 \mathrm{~B}$. Differences were more than twice as great in prism-adapting/prism-removed owls (mean $=0.15$ ) than in control/prism-adapted owls (0.07) and was highly significant ( $p<0.0001$, unpaired Student's $t$ test). Thus, bidirectional CREB regulation was distributed in a patchy, not continuous pattern within rostral ICX.

\section{Model}

We developed a model in which the magnitude of CREB phosphorylation reflected the value of an alignment signal delivered to, or computed by, ICX neurons. This value was determined by comparison of the relative strengths of auditory $(A)$ and visual $(V)$ inputs by an array of comparator cells which, in principle, could be located in OT or ICX. The basis for this model is that the difference between $A$ and $V$ predicts the functional outcome of plasticity: where $V$ is stronger than $A$, a strengthening of inputs occurs, and where $A$ is stronger than $V$, a weakening occurs.

Convergence of $A$ and $V$ information onto the bimodal comparator cells is diagrammed in Figure 9A. For owls whose maps are well aligned (control or prism-adapted), inputs arising from a bimodal stimulus deliver proportionate amounts of $A$ and $V$ drive to each cell in the array. Thus, the computed differences in drive, $V-A$, are approximately zero. In contrast, for owls whose maps are not well aligned (prism-adapting or prism-removed), the differences in drive include both positive and negative values.

To investigate the predictions of the model, we generated a parameter space of $V$ and $A$ values ranging from 0 to 1 in strength, as shown in Figure 9B. Sampling the center wedge of this population, which represents control/prism-adapted owls in which relative values of $A$ and $V$ are on par, generates the unimodal distribution shown in Figure $9 C$, far right panel (top graph above "subtraction"). Sampling the peripheral wedges, which represent prism-adapting/prism-removed owls, generates the bimodal distribution shown in the bottom far right panel. The shapes of these $V-A$ value distributions best match the pCREB/CREB distributions that were found experimentally. Three other potential "comparator rules" were examined: addition, multiplication, and division. The latter rule also generated unimodal and bimodal distribution for control and prism conditions respectively, but the former two rules did not (Fig. 9C). Thus, only those comparison rules that compute the relative magnitude of $A$ and $V$ predicted the empirically determined shape of the pCREB/CREB distributions.

\section{Biochemical diversity of CREB-expressing cells}

To determine the biochemical identity of cells that express CREB, we conducted multiple-label immunohistochemistry. In one experiment, labeling using the CREB antibody along with a mouse IgG1 antibody directed against $\mathrm{CaMKII} \alpha$, which marks a major class of projection neurons in ICX (Rodriguez-Contreras et al. 2005), was accomplished using TSA immunohistochemistry (see Materials and Methods) (Fig. 3). We found that CREB is expressed in $100 \%$ of CaMKII $\alpha$-positive cells $(n=77)$, whereas CaMKII $\alpha$-positive cells represent $5.8 \%$ of CREB-positive cells $(n=1319)$. Same-cell expression of CREB with additional neuroanatomical markers, calbindin, calretinin, and NeuN, revealed that CREB was expressed in $98.6,87.8$, and $98.8 \%$ of cells of these types, respectively (number of cells examined, 72, 41, and 85), and that the percentage of CREB cells positive for these markers was $16.1,16.6$, and $22.0 \%$, respectively (number of CREB cells examined, 440, 217, and 381). Thus, CREB is expressed in a variety of neuronal and non-neuronal cell types in ICX.

\section{Discussion}

We have shown that CREB is bidirectionally regulated during the initial stages of prism learning. This regulation occurs in the population of cells that receive instructive signals and respond with precise reorganization of their auditory spatial receptive fields. All five known properties of the instructive signal were reflected in the empirical profile of CREB regulation: bidirectionality, exclusion from ICC, restriction to rostral ICX, diminishment with adaptation, and patchy topographic distribution (Table 2). These results indicate that cell-specific CREB phosphorylation state encodes the value of the instructive signal. We propose that bidirectional adjustments in CREB-dependent gene expression provide a cell-wide resource that participates in the strengthening or weakening of auditory inputs.

\section{Experience-driven CREB regulation: comparison with other systems}

CREB regulation is readily induced in vitro, by electrical stimulation or pharmacological activation (Kandel, 2001; Lonze and Ginty, 2002). The goals of such studies have been oriented toward elucidating mechanisms of CREB-induced synaptic plasticity. In contrast, less is known regarding CREB regulation driven by naturalistic patterns of sensory experience. Playback of conspecific song induces rapid phosphorylation of CREB in area $\mathrm{X}$-projecting higher vocal center (HVC) neurons of the zebra finch song system. Neither white noise nor the song of an unrelated species (canary) produces a similar effect, indicating that CREB regulation in HVC neurons occurs specifically in response to highly salient natural stimuli (Sakaguchi et al., 1999). These observations suggest an instructive role for CREB; however, the nature of instructive information represented within $\mathrm{HVC}$ is 
obscure (Thompson et al., 2007). In mammalian visual cortex, patterned visual experience causes a transient phosphorylation of ERK (extracellular signal-regulated kinase), leading to cAMP response element (CRE)-mediated gene expression (Cancedda et al., 2003). On this basis, ERK is proposed to act as a molecular readout of patterned activity. The relationship between the structure of that pattern and the spatial distribution of ERK regulation has remained untested. In somatosensory cortex, whisker deprivation causes a large increase in CRE-mediated gene transcription within layer IV of the spared whisker barrel; this is associated with potentiation of responses in layer II/III, a primary postsynaptic target zone (Barth et al., 2000). However, this model of plasticity involves depriving the cortex of a subset of inputs, and the activity signals that guide deprivation-induced plasticity are fundamentally distinct from those that guide instructed learning (Knudsen, 1994).

Of the many studies that have examined the role of CREB in plasticity, ours is unique in two significant respects. First, many basic properties of the instructive signal in the owl auditory localization pathway are known (Table 2). Foremost is that this signal must encode the relative, not total, amounts of $A$ and $V$ activity. By manipulating the magnitude of $A-V$ mismatch in individual owls with distinct histories of prism experience, we disambiguated whether CREB integrates activity level or encodes instructive information.

Experience-driven activation of genes such as c-fos, zenk, and arc reflects the recent level of neural activity (Velho et al., 2005). In principle, CREB could provide a similar role, but that explanation is inconsistent with the current findings. Prisms do not alter the level or pattern of auditory or visually driven activity, only their co-registration. Even if there were unappreciated differences in activity levels between control and adapting owls, learning rules for which output scales with the level of activity, i.e., addition or multiplication of $A$ and $V$ inputs, predict unidirectional, not bidirectional, regulation. In contrast, learning rules for which output encodes the adaptive value, i.e., subtraction or division, predict a bidirectional result (Fig. 9). Thus, the observed patterns of CREB regulation must have been driven by the instructive (adaptive) value of coincident $A$ and $V$ activity.

The second unique aspect of our study is that we quantified CREB regulation at the single-cell level, using ratiometric imaging at individual nuclei (Leutgeb et al., 2005). This highresolution method provided information not apparent from previous studies, most of which examined CREB phosphorylation by analyzing either the number of cells expressing pCREB or CREmediated reporter gene, or by measuring pCREB/CREB in brain tissue homogenates using quantitative immunoblot. The utility of our method is highlighted by the fact that mean pCREB/CREB values were not significantly different for many of the control/ prism-adapting pairs (not shown). Thus, the central and novel findings of our study would have been obscured had the question been tackled using cell counts or quantitative immunoblot.

\section{CREB regulation in rostral ICX}

Instructive information is conveyed to ICX via a sparse topographic projection that originates in the intermediate layers of

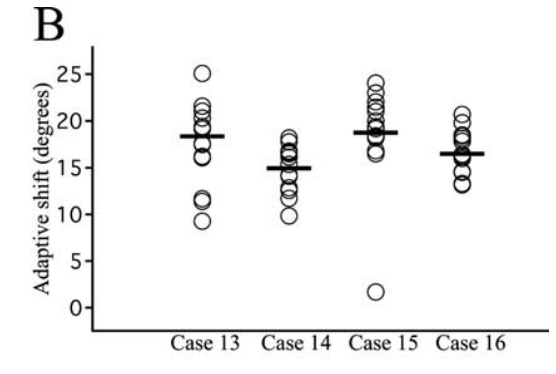

Figure 6. Electrophysiological analysis of prism-adapted owls. $\boldsymbol{A}$, Representative raster plot of auditory responses recorded from the OT of a prism-adapted owl. The gray box indicates time of auditory stimulus. Tick marks represent action potentials. At this site there was a modest level of spontaneous activity and strong evoked responses. Open arrow indicates the Vrf with prisms moved $\left(\mathrm{R} 14^{\circ}\right)$. Filled arrow indicates the Vrf with prisms in place $\left(\mathrm{L} 5^{\circ}\right)$. Responses to sounds presented at the optically displaced circle represents the adaptive shift measured at one recording site. Horimean $=15.0^{\circ}$; ase $15, n=15$, mean $=18.7^{\circ} ;$ case $16, n=15$, mean $=16.5^{\circ}$.

OT (Hyde and Knudsen, 2000). This projection does not penetrate to the ICC core, which is stationed two synapses earlier in the serially organized localization pathway. Hence, the ICC core is physiologically unchanged by prism adaptation (Brainard and Knudsen, 1993). However, it experiences a pattern of ascending auditory drive very similar to that which flows through ICX. These attributes make the ICC core a suitable control structure for the analysis of prism-related effects. In no case was a bimodal distribution of pCREB/CREB values observed in the ICC core (16 owls, control and adapting).

In contrast, bimodal pCREB/CREB distributions were found consistently in ICX, the principal recipient of instructive information. The prism mount used in our experiments suspends the Fresnel lenses $\sim 0.5 \mathrm{~cm}$ in front of the owls' eyes. Frontal visual space, which is represented in rostral ICX, is reliably displaced. In contrast peripheral visual space, represented in caudal ICX, is not. Thus, the $A-V$ mismatches conveyed to rostral ICX should depend on the state of adaptation, whereas those conveyed to caudal ICX should not. This profile was observed for all but one owl, case 15, which exhibited a clear bidirectional effect in both rostral and caudal ICX (Fig. $7 B$; Table 2). One interpretation is that this owl experienced large $A-V$ mismatches across both frontal and peripheral space. This would occur if either the prisms were mounted abnormally close to the eyes, such that the entire visual field was subjected to optical displacement, or if the prism mount had significantly distorted the shape of the facial ruff. Relaxation of the ruff to a natural position after prism removal could transform the spatial pattern of binaural cues in peripheral space, producing $A-V$ mismatches independent of optical displacement. Neither of these physical placement issues (proximity of lens to eye, distortion of ruff) were evident at the time of killing, nor were they routinely measured.

CREB cells in ICX constitute a diverse population, including both neuronal and non-neuronal cell types (see Results). Attempts to parse this population into subsets by costaining with neurochemical markers were confounded by the use of primary antibodies representing common serotypes, mouse monoclonal IgG $1 \alpha$ and rabbit polyclonal antisera, for CREB and pCREB, respectively. In one experiment, triple labeling with a mouse antibody directed against CaMKII was accomplished using an ultrasensitive detection protocol (Fig. 3), but the reacted tissue sections exhibited small anomalies in color separation and therefore were excluded from quantitative analysis. Nuclear volumes 
Prism-adapted

A
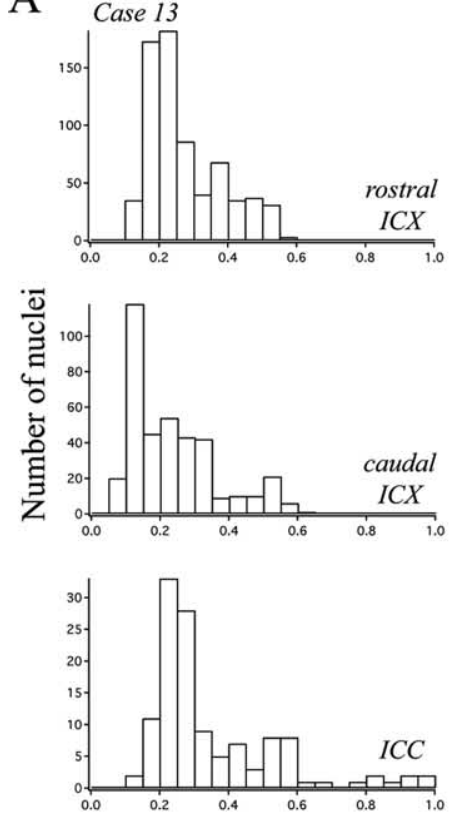
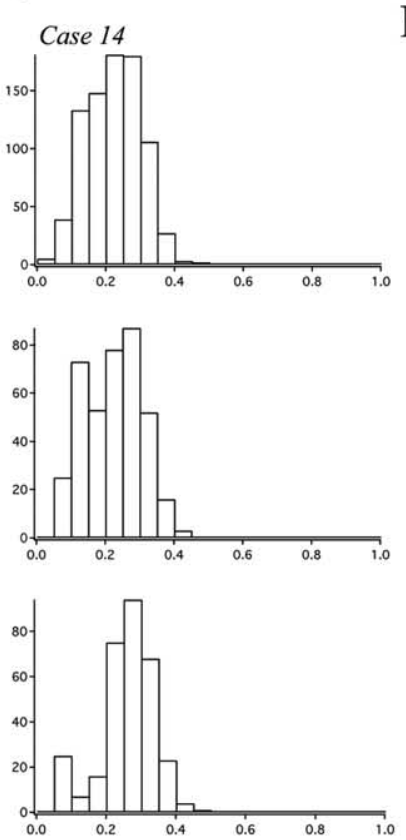

Prism-removed
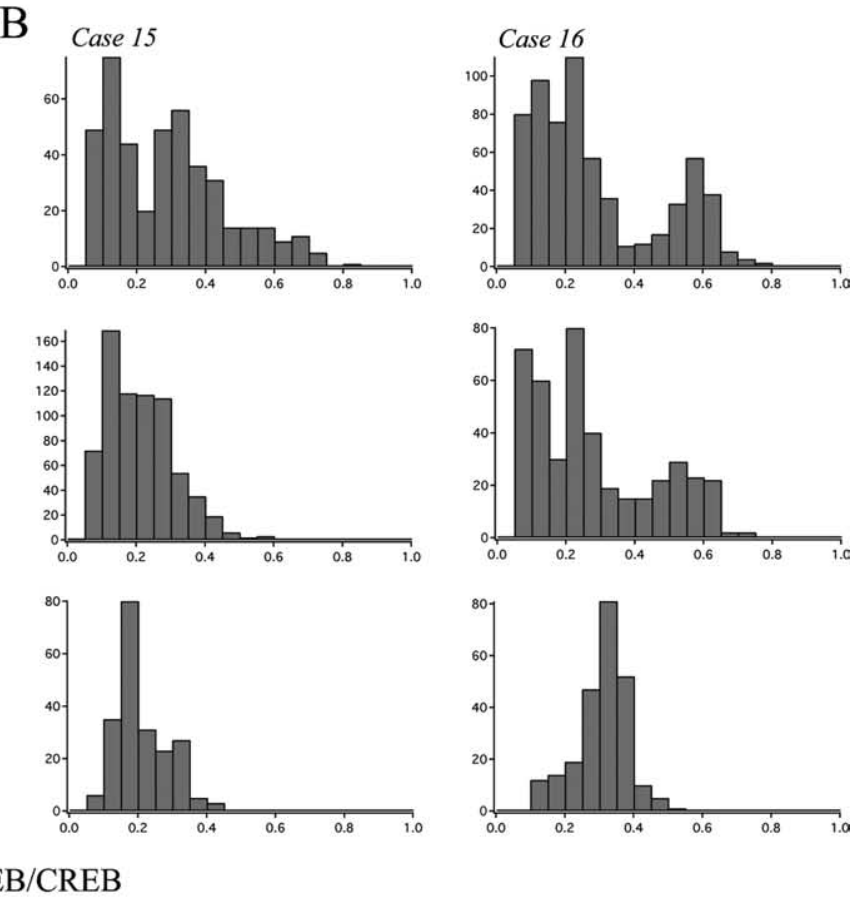

$\mathrm{pCREB} / \mathrm{CREB}$

Figure 7. CREB regulation in prism-adapted and prism-removed owls. $A, B, p C R E B / C R E B$ distributions in rostral ICX, caudal ICX, and ICC core for two prism-adapted owls ( $A$ ) and two prismremoved owls $(\boldsymbol{B})$. All other aspects of this analysis are as described in the Figure 4 legend.
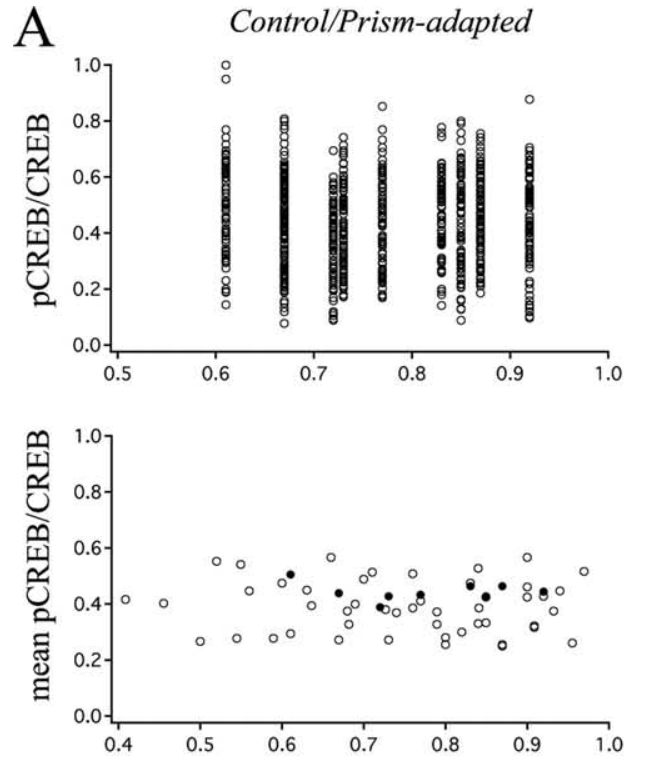

ICX location (rostrocaudal extent)
Prism-adapting/Prism-removed
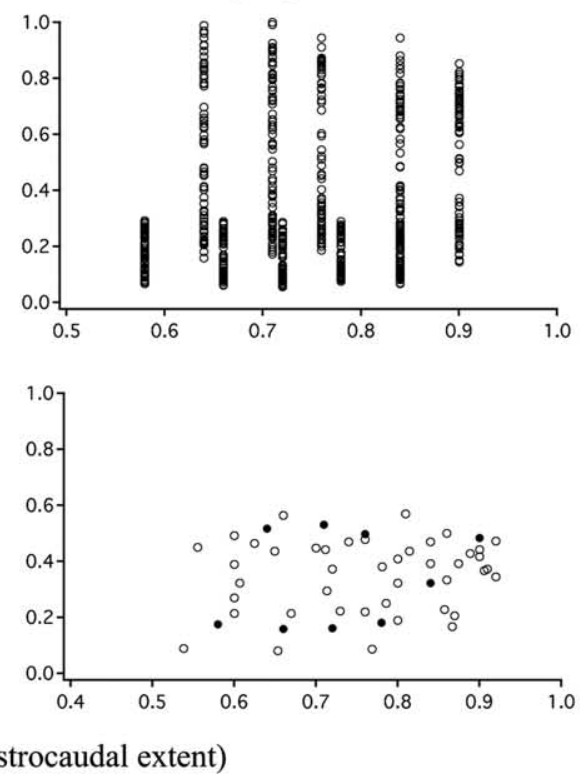

B

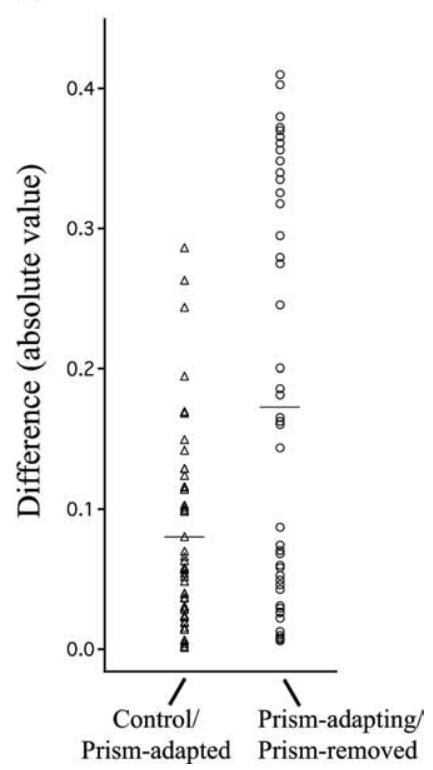

Figure 8. Topographic distribution of CREB regulation. $A$, Top panels, Spatial distribution of $\mathrm{pCREB} / C R E B$ values versus rostrocaudal location in rostral ICX, for a prism-adapted owl experiencing small $A-V$ mismatches (left) and a prism-removed owl experiencing large $A-V$ mismatches (right). Each circle represents a cell nucleus; each vertical line of points represents a single image field. Much less field-to-field variation was observed in the prism-adapted case. Bottom panels, Summary data for all control/prism-adapted owls (left) or prism-adapting/prism-removed owls (right). Each circle represents the mean of each image field. Filled circles correspond to the data presented above. $\boldsymbol{B}$, The difference between the means of neighboring image fields were calculated and plotted for each owl. These difference values reflect the magnitude of field-to-field variation. The mean difference value (horizontal lines) for prism-adapting/prism-removed owls was more than twice as large as that for control/prism-adapted owls $(0.17, n=45$ vs $0.08, n=50 ; p<0.0001$, Student's $t$ test).

were analyzed as one proxy for cell type, and bidirectional regulation was found to be independent of this variable (data not shown). Thus, the prism-induced changes we observed likely occurred in a heterogeneous population of ICX cells. The extent to which this diverse population receives synaptic contacts directly from instructive axons as opposed to indirectly through a polysynaptic pathway within ICX remains an open question.

Several of the comparisons between pCREB/CREB distributions in control versus adapting owls revealed lateral shifts (Figs. 4, 5; Table 1). These shifts were small in magnitude and sporadic 


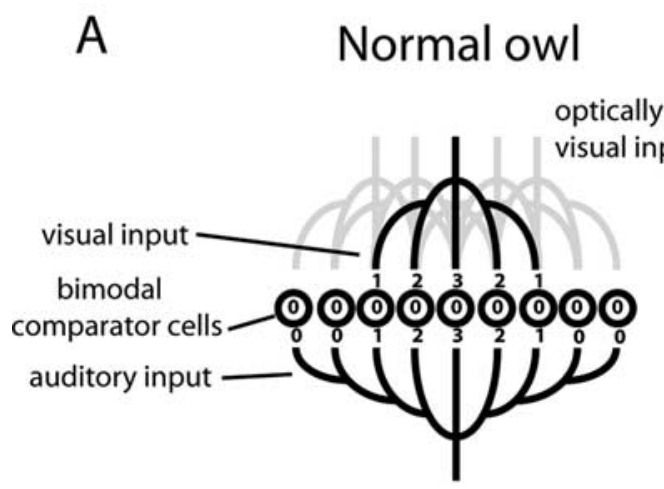

C
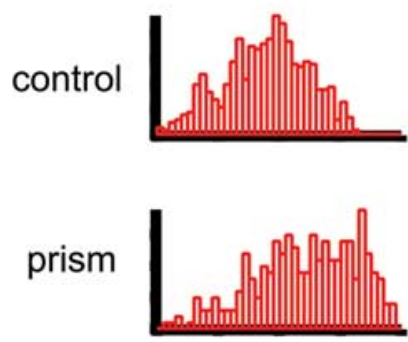

addition
Prism owl
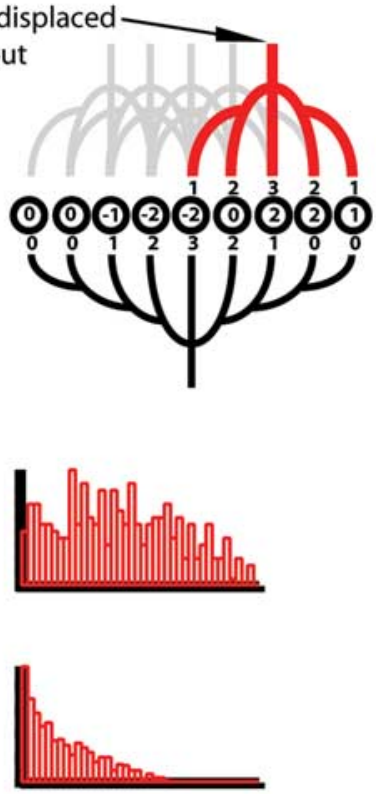

multiplication
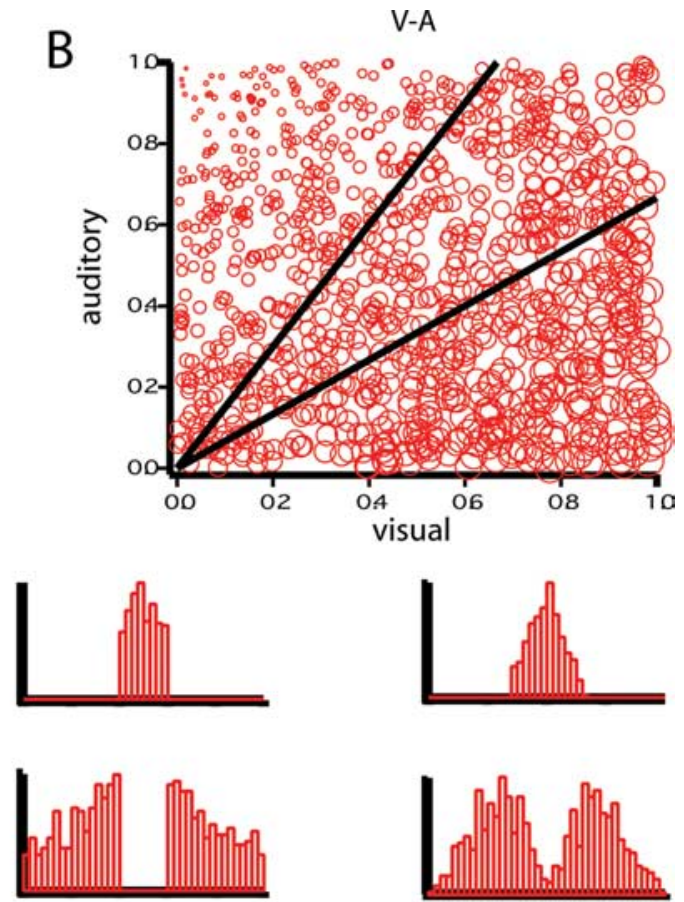

division

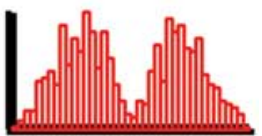

subtraction

Figure 9. $\boldsymbol{A}-\boldsymbol{C}$, Model of CREB as a readout of the magnitude of $A-V$ mismatch. $\boldsymbol{A}$, Schematic diagram illustrating the convergence of auditory and visual activity arising from a discrete bimodal stimulus onto an array of bimodal comparator cells. The activated auditory (bottom) and visual (top) projections are depicted with black lines. Retinotopic projections not activated by the bimodal stimulus are depicted with gray lines. Numbers outside the comparator cells indicate relative strength of drive. Numbers inside the comparator cells indicate the difference between the strengths of auditory and visual drive. In a normal (control or prism-adapted) owl whose maps are well aligned, these differences are nominally zero (left). In a prism-adapting owl, the visual input is displaced (red lines), and therefore, the differences include both positive and negative values (right). B, Scatterplot of 1000 simulated data points that were randomly assigned values from 0 to 1 (uniform distribution) for strengths of $A$ and $V$ drive. The size of each circle scales linearly with the difference, $V-A$. C, Frequency histograms produced by sampling the inner (control) or outer (prism) wedges of the simulation shown in $\boldsymbol{B}$. Histograms were calculated separately for four different comparator rules, $V+A$ (addition), $V^{*} A$ (multiplication), $V /(V+A)$ (division), and $V-A$ (subtraction).

Table 2. Known properties of the instructive signal and effects on CREB

\begin{tabular}{ll}
\hline Instructive signal & CREB effect \\
\hline 1. Conveys positive and negative values & 1. Bidirectional \\
2. Activities in ICX, not ICC & 2 . Observed in ICX, not ICC \\
3. For prisms, large values typically conveyed only to rostral ICX & 3 . Largely restricted to rostral ICX (all cases but one) \\
4. Magnitude diminishes with adaptation, and increases with prism removal & 4 . Eliminated by adaptation and reappears with prism removal \\
5. Activates discrete, not global regions of the space map & 5. Occurs in a patchy, not continuous distribution \\
\hline
\end{tabular}

in direction, although sometimes statistically significant. There was no correspondence between the prevalence or direction of lateral shifts and the four experimental conditions. The most likely explanation for lateral shifts observed between cases is differences in global phosphorylation state, either intrinsic or acquired during perfusion.

\section{Constraints on the model: cell-wide versus}

\section{synapse-specific effects}

We propose that the magnitude of CREB phosphorylation reflects the value of a reward/punishment signal delivered to or computed by ICX neurons. According to this model, the right peak in the bimodal distribution represents cells for which a positive $V-A$ value has been computed, and the left peak, cells for which a negative $V-A$ value has been computed. CREB phosphorylation links this computation to synaptic remodeling because transcription controlled by CREB supports the strengthening and formation of synapses (Barco et al., 2002; Marie et al., 2005), and CREB contributes to both potentiation and depression (Pittenger et al. 2006, Ahn et al. 1999). Because the circuit responsible for the expression of the native space map is not eliminated after prism adaptation (Zheng and Knudsen, 1999) but instead becomes functionally weak, low pCREB/CREB values are not predicted, within this circuit, to lead to synaptic retraction.

CREB-related protein products that strengthen/weaken synapses are presumably distributed as a cell-wide resource, because they originate in the nucleus. However, if all synapses onto a cell for which a positive $V-A$ value was computed were strengthened, synapses not conducive to acquiring the adaptive responses would be strengthened in addition to ones that are (Fig. 9A). This requires the existence of a synaptic tagging mechanism, in which CREB-dependent protein products are used only at input synapses that have been recently marked by activity (Frey and Morris, 1997; Barco et al., 2002). The cytoplasmic polyadenylation element-binding protein (CPEB) has been proposed as one such synaptic mark (Si et al., 2003).

Auditory-visual stimuli occurring in the wake of a behaviorally relevant episode would produce novel patterns of $V-A$ values and, consequently, CREB activation levels, distributed across the 
ICX. If the lifetime of the synaptic tag were longer than the interval between episodes, individual synapses would receive confounding molecular signals. Therefore, the temporal density of behavioral episodes that use CREB to promote synaptic change must be low. One possibility is that an attentional gate restricts the entry or use of instructive information in ICX (Gutfreund et al., 2002). In this scenario, the circuit would "focus" its CREBdependent mechanisms on a subset of experiences, those expected to be particularly useful for calibrating the auditory space map. The paradigm we used, hunting live mice, is known to enhance owls' capacity for prism adaptation (Bergan et al., 2005).

\section{Future experiments}

Bidirectional CREB regulation represents a tool that can be used to monitor arrival of instructive information into ICX. One application is to identify the nature of plasticity failure in adults. Naive adult owls that are passively fed do not adapt to large prismatic displacements, whereas those required to hunt live mice do (Bergan et al., 2005). The plasticity failure of passively fed adults could result from failure to engage attentional mechanisms that gate open the instructive signal, or from agedependent restriction on the capacity of ICX to respond to that signal. Analysis of CREB regulation in actively versus passively fed adults would reveal whether the failure occurs upstream or downstream of the delivery of instructive information.

\section{References}

Ahn S, Ginty DD, Linden DJ (1999) A late phase of cerebellar long-term depression requires activation of CaMKIV and CREB. Neuron 23:559-568.

Barco A, Alarcon JM, Kandel ER (2002) Expression of constitutively active CREB protein facilitates the late phase of long-term potentiation by enhancing synaptic capture. Cell 108:689-703.

Barth AL, McKenna M, Glazewski S, Hill P, Impey S, Storm D, Fox K (2000) Upregulation of cAMP response element-mediated gene expression during experience-dependent plasticity in adult neocortex. J Neurosci 20:4206-4216

Bergan JF, Ro P, Ro D, Knudsen EI (2005) Hunting increases adaptive auditory map plasticity in adult barn owls. J Neurosci 25:9816-9820.

Brainard MS, Knudsen EI (1993) Experience-dependent plasticity in the inferior colliculus: a site for visual calibration of the neural representation of auditory space in the barn owl. J Neurosci 13:4589-4608.

Brainard MS, Knudsen EI (1998) Sensitive periods for visual calibration of the auditory space map in the barn owl optic tectum. J Neurosci 18:3929-3942.

Cancedda L, Putignano E, Impey S, Maffei L, Ratto GM, Pizzorusso T (2003) Patterned vision causes CRE-mediated gene expression in the visual cortex through PKA and ERK. J Neurosci 23:7012-7020.

DeBello WM, Knudsen EI (2004) Multiple sites of adaptive plasticity in the owl's auditory localization pathway. J Neurosci 24:6853-6861.

Frey U, Morris RG (1997) Synaptic tagging and long-term potentiation. Nature 385:533-536.

Gutfreund Y, Zheng W, Knudsen EI (2002) Gated visual input to the central auditory system. Science 297:1556-1559.

Hagiwara M, Alberts A, Brindle P, Meinkoth J, Feramisco J, Deng T, Karin M,
Shenolikar S, Montminy M (1992) Transcriptional attenuation following cAMP induction requires PP-1-mediated dephosphorylation of CREB. Cell 70:105-113.

Hyde PS, Knudsen EI (2000) Topographic projection from the optic tectum to the auditory space map in the inferior colliculus of the barn owl. J Comp Neurol 421:146-160.

Hyde PS, Knudsen EI (2001) A topographic instructive signal guides the adjustment of the auditory space map in the optic tectum. J Neurosci 21:8586-8593.

Hyde PS, Knudsen EI (2002) The optic tectum controls visually guided adaptive plasticity in the owl's auditory space map. Nature 415:73-76.

Kandel ER (2001) The molecular biology of memory storage: a dialog between genes and synapses. Biosci Rep 21:565-611.

Knudsen EI (1994) Supervised learning in the brain. J Neurosci 14:3985-3997.

Knudsen EI (2002) Instructed learning in the auditory localization pathway of the barn owl. Nature 417:322-328.

Konishi M (2003) Coding of auditory space. Annu Rev Neurosci 26:31-55.

Leutgeb JK, Frey JU, Behnisch T (2005) Single cell analysis of activitydependent cAMP-responsive element-binding protein phosphorylation during long-lasting long-term potentiation in area CAl of mature rat hippocampal-organotypic cultures. Neuroscience 131:601-610.

Lonze BE, Ginty DD (2002) Function and regulation of CREB family transcription factors in the nervous system. Neuron 35:605-623.

Marie H, Morishita W, Yu X, Calakos N, Malenka RC (2005) Generation of silent synapses by acute in vivo expression of CaMKIV and CREB. Neuron 45:741-752.

McBride TJ, Rodriguez-Contreras A, Trinh A, Bailey R, Debello WM (2008) Learning drives differential clustering of axo-dendritic contacts in the barn owl auditory system. J Neurosci 28:6960-6973.

Pittenger C, Fasano S, Mazzocchi-Jones D, Dunnett SB, Kandel ER, Brambilla R (2006) Impaired bidirectional synaptic plasticity and procedural memory formation in striatum-specific cAMP response element-binding protein-deficient mice. J Neurosci 26:2808-2813.

Rodriguez-Contreras A, Liu XB, DeBello WM (2005) Axodendritic contacts onto calcium/calmodulin-dependent protein kinase type II-expressing neurons in the barn owl auditory space map. J Neurosci 25:5611-5622.

Sakaguchi H, Wada K, Maekawa M, Watsuji T, Hagiwara M (1999) Songinduced phosphorylation of cAMP response element-binding protein in the songbird brain. J Neurosci 19:3973-3981.

Si K, Giustetto M, Etkin A, Hsu R, Janisiewicz AM, Miniaci MC, Kim JH, Zhu H, Kandel ER (2003) A neuronal isoform of CPEB regulates local protein synthesis and stabilizes synapse-specific long-term facilitation in aplysia. Cell 115:893-904.

Swofford JA, DeBello WM (2007) Transcriptome changes associated with instructed learning in the barn owl auditory localization pathway. Dev Neurobiol 67:1457-1477.

Thompson JA, Wu W, Bertram R, Johnson F (2007) Auditory-dependent vocal recovery in adult male zebra finches is facilitated by lesion of a forebrain pathway that includes the basal ganglia. J Neurosci 27:12308-12320.

Velho TA, Pinaud R, Rodrigues PV, Mello CV (2005) Co-induction of activity-dependent genes in songbirds. Eur J Neurosci 22:1667-1678.

Wagner H, Güntürkün O, Nieder B (2003) Anatomical markers for the subdivisions of the barn owl's inferior-collicular complex and adjacent periand subventricular structures. J Comp Neurol 465:145-159.

Zheng W, Knudsen EI (1999) Functional selection of adaptive auditory space map by GABAA-mediated inhibition. Science 284:962-965. 